\title{
ENFERMERÍA Y GERENCIA DE CASO
}

Santina Nunes Alves Casarin ${ }^{1}$

Tereza Cristina Scatena Villa ${ }^{2}$

Maria Helena Larcher Caliri ${ }^{2}$

Roxana Isabel Cardozo Gonzales ${ }^{3}$

Cinthia Midori Sassaki ${ }^{4}$

Casarin SNA, Villa TCS, Caliri MHL, Gonzales RIC, Sassaki CM. Enfermería y gerenciamento de caso. Rev Latino-am Enfermagem 2001 julho; 9(4):88-90.

El estudio presenta la gerencia de caso como una modalidad de prestación de servicios que surgió en los ambientes de salud americanos y que consiste en la designación de un profesional o equipo de salud responsable por la atención del paciente, durante todo el proceso clínico, coordinando la atención a través de todos los servicios e instituciones que integran el sistema. En la enfermería, esta modalidad fue aceptada para aumentar el compromiso del profesional en la estandarización de las prácticas. Conceptos centrales incluyen análisis, toma de decisiones clínicas y resolución de problemas.

PALABRAS CLAVES: gerencia de caso, gerencia de caso de enfermería, cuidado gerencial

\section{CASE MANAGEMENT AND NURSING}

This study presents case management as a care delivery model which is used in a variety of settings in the United States health care system. It basically designates a specific professional or health care team which is responsible for the care of an individual patient in the whole clinical process by coordinating care throughout services and institutions in the system. In nursing, the model has been accepted to increase the nurse's commitment in the standardization of practice. Central concepts include analysis, clinical decision-making and resolution of problems.

KEY WORDS: case management, nursing case management, managed care

\section{GERENCIAMENTO DE CASO E ENFERMAGEM}

Este estudo apresenta o gerenciamento de caso como uma modalidade de prestação de serviço que surgiu nos ambientes de saúde americano e que consiste na designação de um profissional ou equipe de saúde responsável pela atenção ao paciente durante todo o processo clínico, coordenando a assistência através dos serviços e instituições que integram o sistema. Na enfermagem esta modalidade foi introduzida para aumentar o envolvimento do profissional nas padronizações de práticas. Conceitos centrais incluem análise, decisão clínica e resolução de problemas.

PALAVRAS CHAVE: gerenciamento de caso, gerenciamento de caso de enfermagem, cuidado gerenciado

${ }^{1}$ Enfermera magister en el área de Salud Pública de la Escuela de Enfermería de Ribeirão Preto de la Universidad de São Paulo - Brasil; ${ }^{2}$ Profesor Doctor de la Escuela de Enfermería de Ribeirão Preto de la Universidad de São Paulo, Centro Colaborador de la OMS para el desarrollo de la investigación en enfermería, e-mail: tite@eerp.usp.br; ${ }^{3}$ Enfermera alumna de doctorado en el área de Salud Pública de la Escuela de Enfermería de Ribeirão Preto de la Universidad de São Paulo - Brasil; ${ }^{4}$ Enfermera alumna de maestria en el área de Salud Pública de la Escuela de Enfermería de Ribeirao Preto de la Universidad de São Paulo - Brasil 
INTRODUCCIÓN

Durante los últimos años, en el Brasil y en muchos otros paises, fueron discutido $s$ ampliamente los servicios de salud en relación con su organización y necesidades, con el propósito de encontrar alternativas que orienten su desarrollo y funcionamiento dentro de la nueva realidad socioeconómica y política del mundo contemporáneo.

En los años 90 sucedieron grandes cambios en la forma de ofrecer el cuidado de salud en los Estados Unidos, con el crecimiento del Managed Care Entity (Entidades de cuidado gerencial) que determinan los estándares de atención, normas de tratamiento y las necesidades a ser solucionadas, racionalizando el servicio y la utilización de los prestadores de servicios disponibles.

En ese contexto, apareció una nueva modalidad de prestación de servicio conocido como gerencia de caso, presentándose como un componente de gestión de oferta de utilización de servicios de salud. Esta modalidad es definida como una metodología muy usada por la atención gerencial americana. Consiste en la definición de un único profesional o un equipo de salud que se responsabiliza por la atención del paciente durante todo el proceso clínico y juzga sobre la necesidad de atención y sobre la propiedad de servicios prescritos y recibidos. Ese profesional o equipo de salud, tienen la responsabilidad de coordinar la atención por medio de todos los servicios e instituciones que integran un sistema para determinar el nivel adecuado de prestación de servicios y sí el paciente está cumpliendo el plano de tratamiento ${ }^{(1)}$.

La literatura internacional presenta la gerencia de caso como uno de los modelos de cuidado más reciente adoptado por enfermería y por el sistema de cuidado de salud americano, definido como cuidado integral, continuo, centrado en el paciente y con un equipo multidisciplinario ${ }^{(2)}$.

Utilizando la gerencia de caso se puede optimizar el autocuidado, disminuir l a fragmentación del cuidado, mejorar l a calidad de vida del paciente, disminuir el tiempo de hospitalización, aumentar la satisfacción del paciente y de los profesionales comprometidos y promover el uso de recursos más escasos de forma más efectiva ${ }^{(3)}$.

\section{GERENCIA DE CASO EN ENFERMERÍA}

Enfermeros gerentes de caso son relativamente nuevos en la práctica de la gerencia de caso. La terminología fue adoptada formalmente al inicio de los años 80 . Este modelo de práctica profesional fue aceptado para aumentar el compromiso de la enfermería con la estandarización de las prácticas. Conceptos centrales incluyen análisis, toma de decisiones clínicas y resolución de problemas que constituyen características esenciales para la practica profesional.
A través de la gerencia de caso en enfermería, se equilibra la economía con la calidad del cuidado. Enfermeros son indicados para gerentes de caso debido a su fuerza y conocimiento clínico, habilidad para ofrecer cuidado holístico e abogar en favor del paciente ${ }^{(3)}$.

El enfermero gerente de caso coordina el espectro total del cuidado del paciente en todos sus ambientes, tanto en el hospital como en la comunidad. Ofrece atención particular a las necesidades habituales, defiende al paciente y administra los servicios ${ }^{(4)}$. Así mismo, el puede establecer relaciones efectivas y de colaboración dentro de los departamentos de enfermería, medicina, cuidado domiciliar, cuidado ambulatorio y administración hospitalaria ${ }^{(5)}$.

\section{RESPONSABILIDADES DE ENFERMERÍA EN LA GERENCIA DE CASO}

Éstas dependen del modelo de gerencia de caso adoptado por la institución. Coordinación del plano de cuidados y servicios, es la función más importante en la gerencia de caso, realizado eficientemente, provee un sistema integrado donde todos se benefician. Abogacía del paciente, es realizada cuando los enfermeros ayudan a los pacientes a ordenar sus visiones y preferencias 0 aquellas de un miembro familiar incapacitado para alcanzar autonomía e autodeterminación. A través de la selección/ triage de los casos, frecuentemente el enfermero gerente de caso identifica aquellos pacientes que más se beneficiarían del gerencia de caso y al evaluar y re-evaluar realiza permanentemente evaluaciones del estado físico, apoyo psicosocial y necesidades espirituales del paciente, asimismo considera los recursos financieros disponibles para el caso. Ofrece informaciones para que el paciente y la familia puedan conocer lo que está disponible en relación a los recursos para satisfacer sus necesidades. Elucidar preferencias y prioridades es fundamental. Es frecuentemente el integrador $y$ colaborador del equipo de salud participando de la planeación de la alta lo que requiere la evaluación total de elementos médicos, psicosociales y financieros de cada caso. Para facilitar cambios en los niveles de cuidado, debe estar atento para percibir si el paciente está pronto para cambios de nivel de cuidado e inducir a los médicos a aceptar tales cambios. En el seguimiento después de la alta, su participación puede ser esporádica (en el episodio agudo de la enfermedad), permanente (en todas las fases) o intermitente (cuando necesario). La principal función es negociar e procurar recursos y servicios, actuando como una persona que soluciona problemas. Normalmente persistencia y creatividad producen servicios suficientes para una alta hospitalaria de calidad o para una transición entre los niveles de atención. Ofreciendo el apoyo médico, ejerce el papel de informante frente a cualquier cambio en las condiciones y valores críticos del paciente y sobre su progreso ${ }^{(6)}$. 
Para convertirse en un gerente de caso, según la Case Management Society of America (Sociedad Americana de Gerencia de Caso) es necesario por lo menos tener el grado de bachiller y un año de experiencia de trabajo donde conste el desempeño de actividades de evaluación, comunicación, coordinación de servicios, planeación e implementación de cuidados y abogacía del paciente. El gerente de caso debe estar capacitado para identificar el caso, evaluarlo, identificando problemas, planeando y monitorizando el cuidado, evaluando los resultados de los servicios ofrecidos y reorganizando la atención sí fuese necesario. El gerente de caso tienen la gran responsabilidad de crear recursos frente a las limitaciones del sistema de salud, tiene que realizar funciones más allá de lo que comúnmente realiza un enfermero en el trabajo de rutina ${ }^{(7)}$.

\section{REFERÊNCIAS BIBLIOGRÁFICAS}

1. Mendes EV. Uma agenda para saúde. São Paulo (SP): HUCITEC; 1998.

2. Mumma CM, Nelson A. Models for theory-based practice of rehabilitation nursing. In: Hoeman SP. Rehabilitation nursing: process and application. $2^{\text {nd }}$. ed. St. Louis: MOSBY; 1996.

3. Girard N. The case management model of patient care delivery. AORNJ 1994 setembro; 60(3):403-4, 11.
CONCLUSIÓN

En el contexto actual del sistema de salud son necesarias estrategias que mejoren la atención al paciente con valores más flexibles, innovadores y humanos. En tal sentido el enfermero gerente de caso se constituye en un elemento fundamental para ofrecer mejoras en la atención por la competencia, sentimiento de generosidad, conocimiento clínico, habilidad para ofrecer cuidado holístico y también por la abogacía en favor del paciente. Estas características definirán su papel como gerente de caso en los ambientes de cuidado de salud americano con buenos resultados no solo en el plano asistencial así como también en la práctica de la propia profesión. Consideramos pertinente realizar estudios relacionados con el tema en cuestión, con la finalidad de identificar la utilidad de su aplicación adecuada a la realidad de los servicios de salud y la práctica de enfermería en el Brasil.

4. Rogers M, Riordan J, Swindle D. Community-based nursing case management pays off. Nurs Manage 1991 março; 22(3):30-4.

5. Mcginty MH, Andreoni VM, Quigley MA. Building a Managed Care Approach. Rev Nurs Manage 1993 agosto; 24(8):34.

6. Powell SK. Nursing case management. New York: Lippincott; 1996.

7. Newell M. Using nursing case management to improve health outcomes. Gaithersburg: An Aspen Publication; 1996. 